\title{
ON WITT'S THEOREM IN THE DENUMERABLY INFINITE CASE
}

\author{
BY \\ HERBERT GROSS
}

Introduction. Let $E$ be a finite dimensional vectorspace over a field $k$, and let $\phi$ be a nondegenerate $\varepsilon$-hermitian form, $\phi: E \times E \rightarrow k$, (i.e. $\phi$ is a reflexive sesquilinear form, additive in both arguments and such that for $\lambda, \mu \in k, \phi(x \lambda, y)=\lambda{ }^{J} \phi(x y)$, $\phi(x, y \mu)=\phi(x, y) \mu$ and $\phi(x, y)=\varepsilon \phi(y, x)^{J}$, where $J$ is an involutory antiautomorphism and $\varepsilon$ is in the center of $k$ ). If, in addition, $\phi$ is trace-valued (that is for every $x \in E$ there exists an $\alpha \in k$ such that $\left.\phi(x, x)=\alpha+\varepsilon \alpha^{J}\right)$ then Witt's famous theorem holds: if $F$ is an arbitrary subspace of $E$ then every metric homomorphism $\omega: F \rightarrow E$ which is injective can be extended to a metric automorphism of $E$ [1]. Furthermore, provided $F$ remains finite dimensional, the theorem holds for $E$ of arbitrary dimension.

The following results concerning infinite subspaces of an infinite space $E$ were proved in [4]. Let $E$ be a $k$-vectorspace of denumerable dimension and $\phi$ a nondegenerate, alternate form $\left(^{1}\right)$ (i.e. $\phi(x, x)=0$ for all $x \in E$ ). If $F$ and $F$ are subspaces with $F^{\perp}=(0), F^{\perp}=(0)$ and $\operatorname{dim}(E / F)=\operatorname{dim}(E / F)$ then there exists a metric automorphism of $E$ which maps $F$ onto $F$. Furthermore, there also exists such an automorphism when $F$ and $F$ are closed subspaces $\left(F^{\perp \perp}=F\right)$ with $F \subset F^{\perp}$, $F \subset F^{\perp}, \operatorname{dim} F=\operatorname{dim} F$ and $\operatorname{dim} F^{\perp}=\operatorname{dim} F{ }^{\perp}$ (cf. the explicit normal form in 1.2 below).

In contrast to the finite case, however, prescribed isomorphisms between such subspaces $F$ and $F$ cannot, in general, be extended to automorphisms of $E$.

In the following we shall prove some isomorphism theorems of this kind, concerning both symmetric and alternate forms. They are generalizations of the results in [1] and [4]. The class of subspaces covered by these theorems includes in particular all the closed subspaces $F$ and all the "dense" subspaces $F$. The dimension of $E$ will be kept denumerably infinite throughout.

It is appropriate to mention that this line of attack follows closely Kaplansky's investigations in [4].

\section{Notations and results.}

1.1. Let $E$ and $\bar{E}$ be $k$-vectorspaces, supplied with $\varepsilon$-hermitian forms $\phi$ and $\bar{\phi}$ respectively. A metric (orthogonal) isomorphism is a vector space isomorphism

Presented to the Society, August 18, 1965; received by the editors May 3, 1965.

(1) All the forms discussed here are special instances of $\varepsilon$-hermitian forms. 
$\varphi: E \rightarrow E$ such that $\bar{\phi}(\varphi x, \varphi y)=\phi(x, y)$ for all $x, y \in E$. In particular, two subspaces $F$ and $F$ of $E$ are said to be isomorphic $(F \cong F)$ if there is a metric isomorphism between $F$ and $F$ with respect to the restrictions $\left.\phi\right|_{F}$ and $\left.\phi\right|_{\bar{F}}$ of $\phi$ to $F$ and $F$ respectively. If $F$ is a subspace of $E$ then $F \cap F^{\perp}$ is called the radical (rad $\left.F\right)$ of $F$; $F$ is called semisimple if and only if its radical is trivial; $\phi$ is nondegenerate if and only if $E$ itself is semisimple. As usual, we shall write $\|x\|$ for $\phi(x, x)$. A subspace $F$ of $E$ is said to be closed if $F^{\perp \perp}=F$. If $F$ is closed and $G$ is a finite dimensional subspace, then $F+G$ is closed. $F$ is said to be dense in $E$, if $F^{\perp \perp}=E$, or $F^{\perp}=(0)$.

1.2. We now assume that $\phi$ is trace-valued and that $E$ has denumerably infinite dimension (i.e. $E$ possesses a denumerable algebraic basis). If $F$ is a closed subspace of $E$ with $F \subset F^{\perp}$ then there exists a closed subspace $F^{\prime}$ with $F^{\prime} \subset F^{\prime \perp}$ and $F \cap F^{\prime}=(0)$ such that $F \oplus F^{\prime}$ has an orthogonal supplement in $E, E=\left(F \oplus F^{\prime}\right)$ $\oplus\left(F \oplus F^{\prime}\right)^{\perp}$. Furthermore $F \oplus F^{\prime}$ admits a basis $\left\{e_{i}, e_{i}^{\prime}\right\}_{i \in I}$ with $\phi\left(e_{i}, e_{j}^{\prime}\right)=\delta_{i j}$ and such that $F$ is spanned by the vectors $e_{i}$ and $F^{\prime}$ is spanned by the vectors $e_{i}^{\prime}, i \in I$. In other words, $F \oplus F^{\prime}$ is the orthogonal sum of card $I$ hyperbolic planes $P_{i}=k\left(e_{i}, e_{i}^{\prime}\right),\left(\left\|e_{i}\right\|=\left\|e_{i}^{\prime}\right\|=0, \phi\left(e_{i}, e_{i}^{\prime}\right)=1\right)$. Thus for a closed $F \subset E$ with $F \subset F^{\perp}$ we always have an orthogonal decomposition $E=\left(\bigoplus_{I} P_{i}\right) \oplus G$, $P_{i}=k\left(e_{i}, e_{i}^{\prime}\right)$ a hyperbolic plane, $F=k\left[e_{i}\right]_{i \in I}$.

(A complete proof of this for the alternate case is given in [4]; it carries over without change, cf. $[1$, p. 78$])$.

1.3. Since we shall restrict ourselves to the symmetric case $(\phi(x, y)=\phi(y, x))$ and the alternate case $(\phi(x, x)=0)$, the antiautomorphism $J$ (corresponding to $\phi$ ) is necessarily the identity, hence the underlying field is commutative. If char $k \neq 2$, these forms are trivially trace-valued. When char $k=2$, a trace-valued form is necessarily alternate.

1.4. In the case of a symmetric, nonalternate form we shall make further restrictions on the underlying field, and admit only Kneser fields. A Kneser field $k$ is a commutative nonformally real field of characteristic $\neq 2$ with a finite group $k^{*} / k^{* 2}$ (the multiplicative group of nonzero elements modulo squares). For a detailed discussion of such fields see [3]. If $\phi$ is a nondegenerate symmetric form on the $k$-space $E$ ( $E$ is always of denumerable dimension), and $k$ is a Kneser field, then $E$ possesses an orthonormal basis. In particular, every infinite dimensional subspace of $E$ which is semisimple contains vectors $x$ of arbitrarily prescribed length $\|x\|$. In fact, this is the crucial property we impose in order that the theorems proved below will hold in the symmetric case. The proofs hold without change for any such field. Fields other than Kneser fields for which the alleged property holds are for example the algebraic function fields in one variable over a finite constant field, as follows from the classical results on quadratic forms over such fields in the finite dimensional case (here the order of $k^{*} / k^{* 2}$ is $\aleph_{0}$ ). However our knowledge of examples other than Kneser fields is rather limited. The cases of some important formally real fields will be treated elsewhere. 
1.5. In [2] the following theorem was proved: Let $k$ be a Kneser field and $\phi$ a symmetric, nondegenerate form on $E$. Let $F$ and $\bar{F}$ be subspaces of $E$ with $F^{\perp} \subset F$, $F^{\perp} \subset F$ satisfying the conditions

$$
F \cong F \text { and } \operatorname{dim}(E / F)=\operatorname{dim}(E / F) .
$$

Then there exists an orthogonal isomorphism of $E$ which maps $F$ onto $F$. This theorem will be essential in the proof of Theorem 2 below.

\section{Isomorphism theorems.}

LEMMA 1. Let $k$ be a Kneser field, E semisimple with respect to the symmetric form $\phi$ and of denumerable dimension. Let $F$ and $W$ be subspaces of $E$ with the properties:

(1) $F$ is a finite dimensional subspace spanned by the vectors $f_{1}, f_{2}, \cdots, f_{n}$; and

(2) $W$ is an infinite dimensional semisimple subspace with $W^{\perp} \cap F=(0)$.

Then there exists a vector $x \in E$ such that

(i) $x \in W$,

(ii) $x \notin W \cap F$,

(iii) $\|x\|=\alpha$ for arbitrarily prescribed $\alpha \in k$,

(iv) $\phi\left(x, f_{i}\right)=\beta_{i}$ for arbitrarily prescribed $\beta_{i} \in k(1 \leqq i \leqq n)$.

Proof. (A) Let $F_{1}$ be the subspace of $F$ spanned by $f_{2}, f_{3}, \cdots, f$. By (2) we have $W^{\perp} \cap F_{1}=(0) . F_{1}^{\perp}$ is trivially closed and of finite codimension in $F_{1}^{\perp}+W$. Therefore $F_{1}^{\perp}+W$ is closed and we find $F_{1}^{\perp}+W=\left(F_{1}^{\perp}+W\right)^{\perp \perp}=\left(F_{1}^{\perp \perp} \cap W^{\perp}\right)^{\perp}$ $=\left(F_{1} \cap W^{\perp}\right)^{\perp}=(0)^{\perp}=E$. Hence $\operatorname{dim}\left(W /\left(W \cap F_{1}^{\perp}\right)\right)=\operatorname{dim}\left(\left(W+F_{1}^{\perp}\right) / F_{1}^{\perp}\right)=$ $\operatorname{dim}\left(E / F_{1}^{\perp}\right)=\operatorname{dim} F_{1}$. Thus $W \cap F_{1}^{\perp}$ has an algebraic complement $K$ in $W$, $K \oplus\left(W \cap F_{1}^{\perp}\right)=W$, with $\operatorname{dim} K=\operatorname{dim} F_{1}$.

(B) We next show that $F$ is not perpendicular to $W \cap F_{1}^{\perp}$. For, if $F \subset\left(W \cap F_{1}^{\perp}\right)^{\perp}$ then trivially $F \cap K^{\perp} \subset\left(W \cap F_{1}^{\perp}\right)^{\perp} \cap K^{\perp}=\left[\left(W \cap F_{1}^{\perp}\right)+K\right]^{\perp}=W^{\perp}$. Since $F \cap W^{\perp}=(0)$ (by the assumption of the theorem), $F \cap K^{\perp}=(0)$. On the other hand, $\operatorname{dim}\left(F \cap K^{\perp}\right) \geqq \operatorname{dim} F-\operatorname{dim} K=\operatorname{dim} F-\operatorname{dim} F_{1}=1$, a contradiction. Thus, $F$ is not perpendicular to $W \cap F_{1}^{\perp}$. By the definition of $F_{1}$ we see therefore that $f_{1}$, is not perpendicular to $W \cap F_{1}^{\perp}$. Hence there exists $x_{1} \in W \cap F_{1}^{\perp}$ with

$$
\phi\left(x_{1}, f_{1}\right) \neq 0, \phi\left(x_{1}, f_{i}\right)=0 \quad(1<i \leqq n) .
$$

This procedure can be repeated $n$ times with $F_{j}$, spanned by all the $f_{i}$ with $i \neq j$, in lieu of $F_{1}$. We find in this manner $n$ vectors $x_{1}, x_{2}, \cdots, x_{n}$ with $\phi\left(x_{i}, f_{j}\right)=0$ for $i \neq j$ and $\phi\left(x_{i}, f_{i}\right) \neq 0 \quad(1 \leqq i, j \leqq n)$. The vector $z_{0}=\sum_{i=1}^{n} \beta_{i} \phi\left(x_{i}, f_{i}\right)^{-1} \cdot x_{i}$ satisfies (i) and (iv).

(C) By $1.4 W$ possesses an orthonormal basis. Hence, by the same token $W \cap\left(F+k\left(z_{0}\right)\right)^{\perp}$ contains an infinite dimensional subspace $S$ spanned by an orthonormal basis. Therefore since $k$ is not formally real, $S$ contains a vector $z_{1}$ with $\left\|z_{1}\right\|=\alpha-\left\|z_{0}\right\|$ for arbitrarily prescribed $\alpha \in k$. The vector $z_{2}=z_{0}+z_{1}$ 
now possesses the properties (i), (iii) and (iv). Thus, if $z_{2} \notin W \cap F$ then we are through with our construction. If we should have $z_{2} \in W \cap F$, then we pick a vector $s$ in $S$ with $s \notin F,\|s\|=0$ and $s \perp z_{2}$. The vectors $z_{2}+s$ still has the properties (i), (iii) and (iv) and now we also have $z_{2}+s \notin W \cap F$.

LEMMA 2. Let $k,(E, \phi)$ and the subspace $F$ be as in Lemma 1 . Let $W$ be an infinite dimensional subspace of $E$ such that (1) $W^{\perp}=(0)$ and (2) $W+F \neq E$.

Then there exists a vector $x \in E$ with the properties (iii) and (iv) of Lemma 1 and such that $x \notin W+F$.

Since $W^{\perp}=(0)$ the assumption $W^{\perp} \cap F=(0)$ of Lemma 1 is trivially satisfied and, by starting out with a vector $z_{0}$ with the properties (i) and (iv) (see Step (C)), a proof for Lemma 2 can be found. A complete proof is given in [2].

THEOREM 1. Let $k$ be a Kneser field, E semisimple with respect to the symmetric form $\phi$ and of denumerable dimension. Let $V$ and $\bar{V}$ be infinite dimensional subspaces of $E$ with the properties:

(1) $V^{\perp}$ and $\bar{V}^{\perp}$ are infinite dimensional and semisimple,

(2) $\operatorname{dim}\left(V^{\perp \perp} / V\right)=\operatorname{dim}\left(\bar{V}^{\perp \perp} / \bar{V}\right)$,

(3) $\operatorname{dim}\left(E /\left(V^{\perp}+V^{\perp \perp}\right)\right)=\operatorname{dim}\left(E /\left(\bar{V}^{\perp}+\bar{V}^{\perp \perp}\right)\right)$.

Then there exists an orthogonal automorphism $T: E \rightarrow E$ with $T(V)=\bar{V}$.

Proof. We shall build the automorphism step by step. Assume that we have already constructed four finite dimensional subspaces $F_{i}, F_{i}, G_{i}$ and $G_{\imath}$ with the following properties:

(a) $F_{i} \subset V^{\perp \perp}, \quad F_{i} \subset \bar{V}^{\perp \perp}$,

(b) $G_{i} \cap\left(V^{\perp}+V^{\perp \perp}\right)=G_{i} \cap V^{\perp}, \quad \bar{G}_{i} \cap\left(\bar{V}^{\perp}+\bar{V}^{\perp \perp}\right)=\bar{G}_{i} \cap \bar{V}^{\perp}$.

There exists an orthogonal isomorphism $T_{i}: F_{i} \oplus G_{i} \rightarrow F_{i} \oplus \bar{G}_{i} \operatorname{with}\left({ }^{2}\right)$

(c) $T_{i}\left(F_{i}\right)=F_{i}$,

(d) $T_{i}\left(F_{i} \cap V\right)=F_{i} \cap \bar{V}$,

(e) $T_{i}\left(G_{i}\right)=\bar{G}_{i}$,

(f) $T_{i}\left(G_{i} \cap V^{\perp}\right)=\bar{G}_{i} \cap \bar{V}^{\perp}$.

We are going to enlarge $F_{i} \oplus G_{i}$ and $F_{i} \oplus \bar{G}_{i}$ step by step in such a fashion that each basis vector $e_{n}$ of a fixed basis $\left(e_{n}\right)_{n \geqq 1}$ of $E$ is eventually picked up by some $F_{i} \oplus G_{i}$ and some $F_{j} \oplus \bar{G}_{j}$. In other words, we shall have

$$
E=\bigcup_{i} F_{i} \oplus G_{i}=\bigcup_{i} F_{i} \oplus \bar{G}_{i} \text {. }
$$

Furthermore the construction will be done in such a fashion that at every step conditions (a) through (f) are satisfied: At every step the isomorphism $T_{i}$ is extended to an orthogonal isomorphism $T_{i+1}: F_{i+1} \oplus G_{i+1} \rightarrow \bar{F}_{i+1} \oplus \bar{G}_{+1}$ satisfying the properties (c) through (f) with $i+1$ in lieu of $i$. It is clear by properties (a) through (d), that in this fashion we obtain an orthogonal automorphism $T$ of $E$ which

(2) Notice that by the semisimplicity of $V^{\perp}$ conditions (a) and (b) imply that the sum $F_{i}+G_{i}$ is direct. 
maps $V=\bigcup_{i} V \cap F_{i}$ onto $\bar{V}=\bigcup \bar{V} \cap F_{i}$. We now describe one step in detail. Let $e_{n}$ be the first basis vector not contained in $F_{i} \oplus G_{i}$. We will adjoin it to $F_{i} \oplus G_{i}$ in a suitable way and try to adjoin a matching vector $x$ of $E$ to $\bar{F}_{i} \oplus \bar{G}_{i}$.

Case 1. $e_{n} \notin\left(V^{\perp} \oplus V^{\perp \perp}\right)+\left(F_{i} \oplus G_{i}\right)$. Let us put $W=V^{\perp} \oplus V^{\perp \perp}, F=F_{i} \oplus G_{i}$ and similarly $\bar{W}=\bar{V}^{\perp} \oplus \bar{V}^{\perp \perp}$ and $F=\bar{F}_{i} \oplus \bar{G}_{i}$. Thus $e_{n} \notin W+F$. In particular $W+F \neq E$. Therefore

$$
\operatorname{dim}(F / W \cap F)=\operatorname{dim}(W+F / W)<\operatorname{dim}(E / W)=\operatorname{dim}(E / \bar{W}) .
$$

(The last equality follows from Hypothesis 3 of the theorem.) Furthermore, $\left(V^{\perp} \oplus V^{\perp \perp}\right) \cap\left(F_{i} \oplus G_{i}\right)=F_{i}+\left(V^{\perp} \cap G_{i}\right)$ and $\left(\nabla^{\perp} \oplus \bar{V}^{\perp \perp}\right) \cap\left(F_{i} \oplus G_{i}\right)=F_{i}+\left(\nabla^{\perp} \cap G_{i}\right)$ by (a) and (b), hence, by (c), (e) and (f), we obtain $\operatorname{dim}(F /(W \cap F))=\operatorname{dim}(F /(W \cap F))$. Therefore by using $(\mathrm{I}), \operatorname{dim}((F+\bar{W}) / \bar{W})=\operatorname{dim}(F /(\bar{W} \cap \bar{F}))<\operatorname{dim}(E / \bar{W})$, from which we conclude that $\bar{F}+\bar{W} \neq E$. Now let $f_{1}, \cdots, f_{n}$ be a basis of $F$. By our induction assumption $T_{i} f_{1}, \cdots, T_{i} f_{n}$ is then a basis for $F$. By Lemma 2 there exists a vector $x \in E$ with the properties

$$
x \notin \bar{W}+F,\|x\|=\left\|e_{n}\right\|, \phi\left(x, T_{i} f_{j}\right)=\phi\left(e_{n}, f_{j}\right) \quad(1 \leqq j \leqq n) .
$$

We put $F_{i+1}=F_{i}, G_{i+1}=G_{i} \oplus k\left(e_{n}\right), F_{i+1}=F_{i}$ and $\bar{G}_{i+1}=G_{i} \oplus k(x)$. By virtue of (II) it is clear that $T_{i}$ can be extended to an orthogonal isomorphism $T_{i+1}: F_{i+1} \oplus G_{i+1} \rightarrow F_{i+1} \oplus \bar{G}_{i+1}$ by mapping $e_{n}$ into $x$. Let

$$
z \in G_{i+1} \cap\left(V^{\perp} \oplus V^{\perp \perp}\right),
$$

then $z=\lambda e_{n}+g, \quad g \in G_{i}$. Since $z \in V^{\perp} \oplus V^{\perp \perp}$ we have $\lambda e_{n} \in\left(V^{\perp} \oplus V^{\perp \perp}\right)+G_{i}$. Therefore $\lambda=0$ since $e_{n} \notin\left(V^{\perp} \oplus V^{\perp \perp}\right)+\left(F_{i} \oplus G_{i}\right)$ in the present case. Thus $G_{i+1} \cap\left(V^{\perp} \oplus V^{\perp \perp}\right)=G_{l} \cap\left(V^{\perp} \oplus V^{\perp \perp}\right)$. By the same reasoning $G_{i+1} \cap V^{\perp}$ $=G_{i} \cap V^{\perp}$. Hence, together with (b), $G_{i+1} \cap\left(V^{\perp} \oplus V^{\perp \perp}\right)=G_{i} \cap\left(V^{\perp} \oplus V^{\perp \perp}\right)$ $=G_{i} \cap V^{\perp}=G_{i+1} \cap V^{\perp}$. As $x$ was chosen with $x \notin \bar{W}+F$ we find by the same steps that $\bar{G}_{i+1} \cap\left(\bar{V}^{\perp} \oplus \bar{V}^{\perp \perp}\right)=G_{i+1} \cap \bar{V}^{\perp}$. We see that the intersection property (b) again holds with $i+1$ in place of $i$. Since $G_{i+1} \cap V^{\perp}=G_{i} \cap V^{\perp}$ and $\bar{G}_{i+1} \cap \bar{V}^{\perp}=G_{i} \cap \bar{V}^{\perp}$, the remaining conditions (a) through (f) remain trivially valid after the extension.

Case 2. $e_{n} \in\left(V^{\perp} \oplus V^{\perp \perp}\right)+\left(F_{i} \oplus G_{i}\right)$. We decompose $e_{n}, e_{n}=e_{n}^{\prime}+e_{n}^{\prime \prime}+f+g$ with $e_{n}^{\prime} \in V^{\perp}, e_{n}^{\prime \prime} \in V^{\perp \perp}, f \in F_{i}, g \in G_{i}$. In this case we shall adjoin $e_{n}^{\prime}$ and $e_{n}^{\prime \prime}$ to $G_{i}$ and $F_{i}$ respectively in two successive steps. Assuming that $e_{n}^{\prime}$ is not already an element of $G_{i}$ we first adjoin $e_{n}^{\prime}$ to $G_{i}$ and try to enlarge $G_{i}$ by a suitable match. By the semisimplicity of $\bar{V}^{\perp},\left(\bar{V}^{\perp} \cap \bar{V}^{\perp \perp}=(0)\right)$, and by (b) it follows that $\bar{V}^{\perp \perp} \cap G_{i}=(0)$. Let $g_{1}, g_{2}, \cdots, g_{m}$ be a basis of $G_{i}$. By (e), $T_{i} g_{1}, \cdots, T_{i} g_{m}$ is a basis for $G_{i}$. By Lemma 1 (with $\nabla^{\perp}$ and $G_{i}$ in the roles of $W$ and $F$ respectively) there exists a vector $y \in E$ with the properties

$$
y \in \bar{V}, y \notin \bar{\nabla} \cap G_{i},\|y\|=\left\|e_{n}^{\prime}\right\|, \phi\left(y, T_{i} g_{j}\right)=\phi\left(e_{n}^{\prime}, g_{j}\right) \quad(1 \leqq j \leqq m)
$$


Since $e_{n}^{\prime} \in V^{\perp}$ and $y \in \bar{V}^{\perp}$ we have by (a) that $e_{n}^{\prime} \perp F_{i}$ and $y \perp F_{i}$. Using this together with (III) we can extend $T_{i}: F_{i} \oplus G_{i} \rightarrow F_{i} \oplus \bar{G}_{i}$ to an orthogonal isomorphism $T_{i+1}: F_{i} \oplus G_{i} \oplus k\left(e_{n}^{\prime}\right) \rightarrow F_{i} \oplus \bar{G}_{i} \oplus k(y)$ simply by mapping $e_{n}^{\prime}$ into $y$. Setting $F_{i+1}=F_{i}, \quad G_{i+1}=G_{i}+k\left(e_{n}^{\prime}\right), \quad F_{i+1}=F_{i}$ and $\bar{G}_{i+1}=\bar{G}_{i} \oplus k(y)$ we find $G_{i+1} \cap\left(V^{\perp} \oplus V^{\perp \perp}\right)=G_{i} \cap\left(V^{\perp} \oplus V^{\perp \perp}\right) \oplus k\left(e_{n}^{\prime}\right)$ and $G_{i+1} \cap V^{\perp}=\left(G_{i} \cap V^{\perp}\right)$ $\oplus k\left(e_{n}^{\prime}\right)$. By (b) we obtain again $G_{i+1} \cap\left(V^{\perp} \oplus V^{\perp \perp}\right)=G_{i+1} \cap V^{\perp}$ and similarly for $\bar{G}_{i+1}$. Furthermore, by the definition of the extension $T_{i+1}$ we have $T_{i+1}\left(G_{i+1} \cap V^{\perp}\right)=T_{i}\left(G_{i} \cap V^{\perp}\right)+T_{i+1}\left(k\left(e_{n}^{\prime}\right)\right)=\left(\bar{G}_{i} \cap \bar{V}^{\perp}\right) \oplus k(y)=\bar{G}_{i+1} \cap \bar{V}^{\perp}$. Thus, (b) and (f) remain valid after the extension. For the rest of the conditions this is trivially the case.

There remains $e_{n}^{\prime \prime}$ to be adjoined to $F_{i+1}$. We recall that $e_{n}^{\prime \prime} \in V^{\perp \perp}$. Let us first consider the case where $e_{n}^{\prime \prime} \in V$ (assuming that $e_{n}^{\prime \prime}$ is not in $F_{i} \oplus G_{i}$ already). Let $H_{1}$ be an algebraic complement of $G_{i+1} \cap V^{\perp}$ in $G_{i+1}$. Since $T_{i+1}\left(G_{i+1} \cap V^{\perp}\right)$ $=\bar{G}_{i+1} \cap \bar{V}^{\perp}$ by (f), the image $\bar{H}_{1}=T_{i+1}\left(H_{1}\right)$ is an algebraic complement of $\bar{G}_{i+1} \cap \bar{V}^{\perp}$ in $\bar{G}_{i+1}$. We next convince ourselves of the fact that

$$
V^{\perp} \cap\left(F_{i+1} \oplus H_{1}\right)=(0), \bar{V}^{\perp} \cap\left(F_{i+1} \oplus \bar{H}_{1}\right)=(0) .
$$

For, if $x \in V^{\perp} \cap\left(F_{i+1} \oplus H_{1}\right)$ with $x=f+g$ then $g \in V^{\perp}+F_{i+1} \subset V^{\perp} \oplus V^{\perp \perp}$ by (a). So by (b) $g \in\left(V^{\perp} \oplus V^{\perp \perp}\right) \cap G_{i+1}=V^{\perp} \cap G_{i+1}$. Consequently $g=0$ since $g$ also belongs to the complementary space $H_{1}$. Furthermore $f=0$ by (a) and the fact that $V^{\perp}$ is semisimple $\left(V^{\perp} \cap V^{\perp \perp}=(0)\right)$.

By virtue of (IV) we may apply Lemma 1 (with $\bar{V}$ and $F_{i+1} \oplus \bar{H}_{1}$ in the roles of $W$ and $F$ ). We choose a basis $l_{1}, \cdots, l_{r}$ of $F_{i+1} \oplus H_{1} . T_{i+1} l_{1}, \cdots, T_{i+1} l_{r}$ is then a basis for $F_{i+1} \oplus \bar{H}_{1}$. There exists a vector $z \in E$ with the properties

$$
z \in \bar{V}, z \notin \bar{V} \cap\left(F_{i+1} \oplus \bar{H}_{1}\right),\|z\|=\left\|e_{n}^{\prime \prime}\right\|, \phi\left(z, T_{i+1} l_{j}\right)=\phi\left(e_{n}^{\prime \prime}, l_{j}\right)(1 \leqq j \leqq r)
$$

We set $F_{i+2}=F_{i+1} \oplus k\left(e_{n}^{\prime \prime}\right), G_{i+2}=G_{i+1}, F_{i+2}=F_{i+1} \oplus k(z)$ and $\bar{G}_{i+2}=\bar{G}_{i+1}$. We have

$$
G_{i+1}=\left(G_{i+1} \cap V^{\perp}\right) \oplus H_{1} \text { and } \bar{G}_{i+1}=\left(\bar{G}_{i+1} \cap \bar{V}^{\perp}\right) \oplus \bar{H}_{1} .
$$

Furthermore by (a) we have $e_{n}^{\prime \prime} \perp G_{i+1} \cap V^{\perp}$ and $z \perp G_{i+1} \cap \bar{V}^{\perp}$. Thus, by (V) and (VI) we extend $T_{i+1}$ to an orthogonal isomorphism $T_{i+2}: F_{i+2} \oplus G_{i+2} \rightarrow F_{i+2}$ $\oplus \vec{G}_{i+2}$ by mapping $e_{n}^{\prime \prime}$ into $z$. Since $e_{n}^{\prime \prime} \in V$ and $z \in V$ we find $F_{i+2} \cap V=\left(F_{i+1} \cap V\right)$ $+k\left(e_{n}^{\prime \prime}\right)$ etc. and conditions (a) through (f) remain trivially valid after the extension. The case where $e_{n}^{\prime \prime} \in V^{\perp \perp}$ but $e_{n}^{\prime \prime} \notin V$ remains to be discussed. We have $V+F_{i+1} \neq V^{\perp \perp}$ since $e_{n}^{\prime \prime} \notin V+F_{i+1}$ by assumption. Therefore,

$$
\operatorname{dim}\left(F_{i+1} /\left(V \cap F_{i+1}\right)\right)=\operatorname{dim}\left(\left(F_{i+1}+V\right) / V\right)<\operatorname{dim}\left(V^{\perp \perp} / V\right)=\operatorname{dim}\left(\bar{V}^{\perp \perp} / \bar{V}\right)
$$

where the last equality is obtained by the assumption 2 of the theorem. By (c) and (d) we obtain $\operatorname{dim}\left(F_{i+1} /\left(V \cap F_{i+1}\right)\right)=\operatorname{dim}\left(F_{i+1} /\left(\bar{V} \cap F_{i+1}\right)\right)$ hence

$$
\operatorname{dim}\left(\left(F_{i+1}+\bar{V}\right) / \bar{V}\right)=\operatorname{dim}\left(F_{i+1} /\left(\bar{V} \cap F_{i+1}\right)\right)<\operatorname{dim}\left(\bar{V}^{\perp \perp} / \bar{V}\right)
$$


and we learn that $\bar{V}+\bar{F}_{i+1} \neq \bar{V}^{\perp \perp}$. Let $\bar{V}^{\perp_{0}}$ be the orthogonal complement of $\bar{V}$ in $\bar{V}^{\perp \perp}$. Since $\bar{V}^{\perp}$ is semisimple, we have $\bar{V}^{\perp_{0}}=(0)$. Hence we can apply Lemma 2 to the semisimple vectorspace $\left(\bar{V}^{\perp \perp},\left.\phi\right|_{V^{\perp \perp}}\right), \bar{V}$ and $F_{i+1}$ in the roles of $W$ and $F$ respectively. We choose a basis $f_{1}, \cdots, f_{m}$ for $F_{i+1}$ and find a vector $z_{0} \in V^{\perp \perp}$ with the properties

$$
z_{0} \notin \bar{V}+F_{i+1},\left\|z_{0}\right\|=\left\|e_{n}^{\prime \prime}\right\| \text { and } \phi\left(z_{0}, T_{i+1} f_{j}\right)=\phi\left(e_{n}^{\prime \prime}, f_{j}\right) \quad(1 \leqq j \leqq m) .
$$

Then let $g_{1}, \cdots, g_{s}$ be a basis of $H_{1}$ where $H_{1}$ is again an algebraic complement of $G_{i+1} \cap V^{\perp}$ in $G_{i+1} . T_{i+1} g_{1}, \cdots, T_{i+1} g_{s}$ is then the basis of an algebraic complement $\bar{H}_{1}$ of $\bar{G}_{i+1} \cap \bar{V}^{\perp}$ in $\bar{G}_{i+1}$. One proves as before that

$$
\bar{V}^{\perp} \cap\left(F_{i+1} \oplus \bar{H}_{1} \oplus k\left(z_{0}\right)\right)=(0) .
$$

Once more we apply Lemma 1 (with $\bar{V}$ and $F_{i+1} \oplus \bar{H}_{1} \oplus k\left(z_{0}\right)$ in the roles of $W$ and $F$ respectively) and find a vector $z_{00} \in E$ with

$$
\begin{array}{r}
z_{00} \in \bar{V}, z_{00} \notin \bar{V} \cap F_{i+1} \oplus \bar{H}_{1} \oplus k\left(z_{0}\right)(3),\left\|z_{00}\right\|=0 . \\
\phi\left(z_{00}, z_{0}\right)=0, \phi\left(z_{00}, T_{i+1} f_{j}\right)=0, \phi\left(z_{00}, T_{i+1} g_{l}\right)=\phi\left(e_{n}^{\prime \prime}, g_{l}\right)-\phi\left(z_{0}, T_{i+1} g_{l}\right) \\
(1 \leqq j \leqq m, 1 \leqq l \leqq s) .
\end{array}
$$

We set $z=z_{0}+z_{00}$ and by (VII) and (VIII) we find that

$$
\begin{gathered}
z \in \bar{V}^{\perp \perp}, z \notin \bar{V}+F_{i+1}+\bar{G}_{i+1},\|z\|=\left\|e_{n}^{\prime \prime}\right\|, \\
\phi\left(z, T_{i+1} f_{j}\right)=\phi\left(e_{n}^{\prime \prime}, f_{j}\right) \text { and } \phi\left(z, T_{i+1} g_{l}\right)=\phi\left(e_{n}^{\prime \prime}, g_{l}\right) .
\end{gathered}
$$

We put $F_{i+2}=F_{i+1} \oplus k\left(e_{n}^{\prime \prime}\right), G_{2+1}=G_{i+1}, F_{i+2}=F_{i+1} \oplus k(z)$ and $\bar{G}_{i+2}=\bar{G}_{i+1}$. As before we have $e_{n}^{\prime \prime} \perp G_{i+1} \cap V^{\perp}, z \perp G_{i+1} \cap \bar{V}^{\perp}$.

From (IX) we see that $T_{i+1}$ can again be extended to $T_{i+2}: F_{i+2} \oplus G_{i+2} \rightarrow F_{i+2}$ $\oplus \bar{G}_{i+2}$. In this case we have $F_{i+2} \cap V=F_{i+1} \cap V$ and $F_{i+2} \cap \bar{V}=\bar{F}_{i+1} \cap \bar{V}$. All the conditions (a) through (f) remain trivially satisfied after this extension.

This completes the proof of our theorem. Summarizing, we remark that we have seen how to enlarge $F_{i} \oplus G_{i}$ and $F_{i} \oplus G_{i}$ by at least one dimension in such a fashion that (a) through (f) remain valid after the extension. Furthermore, $F_{i} \oplus G_{i}$ has been enlarged by adjunction of a prescribed basis vector. It is clear that in this fashion we can arrive at $\bigcup_{i} F_{i} \oplus G_{i}=E$. However, in order to make sure that $\bigcup F_{i} \oplus \bar{G}_{i}=E$ also, one has to alternate between $F_{\imath} \oplus G_{i}$ and $F_{i} \oplus \bar{G}_{i}$. In the next step the first basis vector not contained in $F_{i+1} \oplus \bar{G}_{i+1}$ is added to $F_{i+1} \oplus G_{i+1}$ and $F_{i+1} \oplus G_{i+1}$ this time is enlarged by a suitable match.

We are now in a position to prove our main theorem. It covers the class of subspaces $H \subset E$ for which $\operatorname{rad} H=\operatorname{rad}\left(H^{\perp}\right)$. In particular, this condition is satisfied by closed subspaces. Examples of spaces which are not closed and which satisfy this condition are the dense subspaces since every space $H$ with $H^{\perp} \subset H$ falls into our category.

(3) This property will not be needed. 
THEOREM 2. Let $k$ be a Kneser field(4), E semisimple with respect to the symmetric form $\phi$ and of denumerable dimension. Let $H$ and $\bar{H}$ be isomorphic subspaces with $\operatorname{rad} H=\operatorname{rad}\left(H^{\perp}\right)$ and $\operatorname{rad} \bar{H}=\operatorname{rad}\left(\bar{H}^{\perp}\right)$ satisfying the following properties:

(i) $H^{\perp} \cong \bar{H}^{\perp}$,

(ii) $\operatorname{dim}\left(\left(H^{\perp}+H^{\perp \perp}\right) /\left(H^{\perp}+H\right)\right)=\operatorname{dim}\left(\left(\bar{H}^{\perp}+\bar{H}^{\perp \perp}\right) /\left(H^{\perp}+\bar{H}\right)\right)$,

(iii) $\operatorname{dim}\left((\operatorname{rad} H)^{\perp} /\left(H^{\perp}+H^{\perp \perp}\right)\right)=\operatorname{dim}\left((\operatorname{rad} \bar{H})^{\perp} /\left(\bar{H}^{\perp}+\bar{H}^{\perp \perp}\right)\right)$.

Then there exists an orthogonal automorphism of $E$ which maps $H$ onto $\bar{H}$.

Conditions (i), (ii) and (iii) are trivially necessary for such an automorphism to exist. Furthermore, condition (i) can be replaced (under the present conditions) by simpler conditions on dimensions, namely

(i') $\operatorname{dim}\left(\left(H+H^{\perp}\right) / H\right)=\operatorname{dim}\left(\left(\bar{H}+\bar{H}^{\perp}\right) / \bar{H}\right)$ provided that this dimension is infinite. If $\operatorname{dim}\left(\left(H+H^{\perp}\right) / H\right)$ is finite, the (necessary) condition that $H^{\perp}$ and $\bar{H}^{\perp}$ be isomorphic is not implied by (i') and the other conditions, unless $k$ is "perfect" (i.e. the order of $k^{*} / k^{* 2}$ is 1 ). For otherwise, there are at least two nonisomorphic 1-dimensional $k$-spaces $L_{1}$ and $L_{2}$. The external sums $E \oplus L_{1}$ and $E \oplus L_{2}$ possess orthonormal bases, hence $E \oplus L_{1}$ $\cong E \oplus L_{2}$ and therefore they provide a simple counterexample.

Proof. (a) Let $H_{0}$ be an algebraic complement of $\operatorname{rad} H$ in $H$ and $H_{1}$ an algebraic complement of $\operatorname{rad} H$ in $H^{\perp}$. We then have the orthogonal decomposition $H+H^{\perp}=H_{0} \oplus \operatorname{rad}(H) \oplus H_{1}$. Furthermore let $L$ be an algebraic complement of $H$ in $H^{\perp \perp}$. Since $\operatorname{rad} H=\operatorname{rad}\left(H^{\perp}\right)$, we find that $\left(H+H^{\perp}\right) \cap H^{\perp \perp}=H$. In particular, $L \cap H=(0)$ and we have the direct decomposition

$$
H+H^{\perp}+H^{\perp \perp}=H_{0} \oplus \operatorname{rad}(H) \oplus H_{1} \oplus L .
$$

(b) Since $\operatorname{rad} H=\operatorname{rad}\left(H^{\perp}\right)=\left(H+H^{\perp}\right)^{\perp}$ we see that $\operatorname{rad} H$ is a closed space. Therefore by 1.2 there exists a subspace $R$ with $R \cap \operatorname{rad} H=(0)$ and a decomposition $E=(\operatorname{rad}(H) \oplus R) \oplus E_{0}$ with $E_{0} \perp \operatorname{rad}(H)+R$, where $\operatorname{rad}(H) \oplus R$ is an orthogonal sum of hyperbolic planes:

$$
\begin{aligned}
\operatorname{rad}(H) \oplus R & =\bigoplus_{i \in I} P_{i}, \\
\operatorname{card} I & =\operatorname{dim}(\operatorname{rad} H), \\
P_{i} & =k\left(e_{i}, e_{i}^{\prime}\right) \text { with } \operatorname{rad} H=k\left[e_{i}\right]_{i \in I}, R=k\left[e_{i}^{\prime}\right]_{i \in I}
\end{aligned}
$$

There exist subspaces $H_{0}^{*}, H_{1}^{*}, L^{*}$ all orthogonal to $R$ such that

$$
\begin{aligned}
& \operatorname{rad}(H) \oplus H_{0}^{*}=\operatorname{rad}(H) \oplus H_{0}(=H), \\
& \operatorname{rad}(H) \oplus H_{1}^{*}=\operatorname{rad}(H) \oplus H_{1}\left(=H^{\perp}\right), \\
& \operatorname{rad}(H) \oplus L^{*}=\operatorname{rad}(H) \oplus L\left(\operatorname{so} \operatorname{rad}(H) \oplus H_{0}^{*} \oplus L^{*}=H^{\perp \perp}\right) .
\end{aligned}
$$

(4) Cf. the discussion at the end of 1.4. 
Since $\operatorname{rad} H \perp H_{0}^{*}, H_{1}^{*}, L^{*}$ we have $H_{0}^{*}, H_{1}^{*}, L^{*} \subset E_{0}$. Without loss of generality we may assume that $H_{0}^{*}$ was chosen for $H_{0}$ at the beginning and similarly for $H_{1}^{*}$ and $L_{1}^{*}$. Hence we shall drop the asterisks. Thus, if $K$ is some algebraic complement of $H_{0} \oplus H_{1} \oplus L\left(=H_{0}^{*} \oplus H_{1}^{*} \oplus L_{1}^{*}\right)$ in $E_{0}$ we have the decompositions

(2) $E=(\operatorname{rad}(H) \oplus R) \oplus E_{0}$ with $E_{0} \perp(\operatorname{rad}(H)+R)$ and $E_{0}=H_{0} \oplus H_{1} \oplus L \oplus K$.

We find $(\operatorname{rad} H)^{\perp}=\operatorname{rad}(H) \oplus E_{0}$. This gives

$$
\operatorname{dim} K=\operatorname{dim}\left((\operatorname{rad} H)^{\perp} /\left(H^{\perp}+H^{\perp \perp}\right)\right) \text {. }
$$

We also read off

$$
\operatorname{dim} L=\operatorname{dim}\left(\left(H^{\perp}+H^{\perp \perp}\right) /\left(H^{\perp}+H\right)\right) .
$$

With respect to the subspace $\bar{H}$ we have a similar decomposition

$$
\begin{gathered}
E=(\operatorname{rad}(\bar{H}) \oplus \bar{R}) \oplus E_{0} \text { with } \bar{E}_{0} \perp(\operatorname{rad}(\bar{H}) \oplus \bar{R}) \text { and } \operatorname{rad}(\bar{H}) \oplus R=\bigoplus_{i \in \bar{I}} \bar{P}_{i}, \\
\bar{E}_{0}=\bar{H}_{0} \oplus \bar{H}_{1} \oplus \bar{L} \oplus R
\end{gathered}
$$

(with $\bar{P}_{i}, \bar{H}_{0}, \bar{H}_{1}, L, R$ analogous objects to those above).

(c) By assumption $H$ and $\bar{H}$ are isomorphic, in particular $\operatorname{dim}(\operatorname{rad} \bar{H})=\operatorname{dim}(\operatorname{rad} H)$ since a radical is mapped on a radical under any isomorphism. We therefore have the same number of hyperbolic planes in the decompositions (1) and (5) of $\operatorname{rad}(H) \oplus R$ and $\operatorname{rad}(\bar{H}) \oplus \bar{R}$ respectively: card $I=$ card $\bar{I}$. Hence, by linear extension of the index map which sends the basis vectors $e_{i}, e_{i}^{\prime}$ of $P_{i}$ into the basis vectors $\bar{e}_{i}, \bar{e}_{i}^{\prime}$ of $\bar{P}_{i}$ respectively, we obtain an orthogonal isomorphism

$$
T_{1}: \operatorname{rad}(H) \oplus R \rightarrow \operatorname{rad}(\bar{H}) \oplus \bar{R} \operatorname{with} \mid T_{1}(\operatorname{rad} H)=\operatorname{rad} \bar{H} .
$$

Similarly, $H \cong \bar{H}$ implies that any two algebraic complements of (the totally isotropic spaces) $\operatorname{rad} H$ and $\operatorname{rad} \bar{H}$ in $H$ and $\bar{H}$ respectively, are isomorphic. In particular

$$
H_{0} \cong \bar{H}_{0} \text {. }
$$

Since furthermore $H^{\perp} \cong \bar{H}^{\perp}$ by assumption of the theorem and since $\operatorname{rad}\left(H^{\perp}\right)$ $=\operatorname{rad} H$ etc., by the same token,

$$
H_{1} \cong \bar{H}_{1} .
$$

By assumptions (ii) and (iii) of the theorem and by (3) and (4) (and their analogues for $L$ and $R$ ) we obtain

$$
\operatorname{dim} L=\operatorname{dim} L \text { and } \operatorname{dim} K=\operatorname{dim} R .
$$

(d) We now consider various cases which may arise and which have to be treated separately.

Case 1. $H_{0}$ and $H_{1}$ are both finite dimensional. Since we have seen that 
$\operatorname{rad} H$ is closed, the same is true for $H=\operatorname{rad}(H) \oplus H_{0}$ and hence for $H^{\perp \perp}+H^{\perp}=H+H^{\perp}=H \oplus H_{1}(1.1)$. Therefore $H^{\perp}+H^{\perp \perp}=\left(H^{\perp}+H^{\perp \perp}\right)^{\perp \perp}$ $=\left(H^{\perp \perp} \cap H^{\perp}\right)^{\perp}=(H \cap H)^{\perp \perp}=(\operatorname{rad} H)^{\perp}$. Hence $L=K=\bar{L}=K=(0)$ by (3), (4) and (9). $E$ decomposes as follows:

$$
E=(\operatorname{rad}(H) \oplus R) \oplus\left(H_{0} \oplus H_{1}\right) \text { and } E=(\operatorname{rad}(\bar{H}) \oplus \bar{R}) \oplus\left(\bar{H}_{0} \oplus \bar{H}_{1}\right) .
$$

Since $H_{0} \oplus H_{1}$ and $\bar{H}_{0} \oplus \bar{H}_{1}$ are orthogonal summands in these decompositions, isomorphisms (6), (7) and (8) define an automorphism of $E$ which maps $H=\operatorname{rad}(H) \oplus H_{0} \quad$ on $\quad \bar{H}=\operatorname{rad}(\bar{H}) \oplus \bar{H} \quad\left(H_{0} \perp H_{1}, \bar{H}_{0} \perp \bar{H}_{1} !\right)$.

We are left with the case where at least one of $H_{0}$ and $H_{1}$ is infinite dimensional. In particular, the semisimple spaces $E_{0}$ and $\bar{E}_{0}$ are then both infinite dimensional and so possess orthonormal bases (1.4). In other words, we have

$$
E_{0} \cong \bar{E}_{0} \text {. }
$$

Case 2. $H_{0}$ is finite dimensional. Since $H_{0}$ is also semisimple, $H_{0}$ has an orthogonal supplement $E_{1}$ in $E_{0}$. The same is the case for $\bar{H}_{0}$ by virtue of (7). So

$$
E_{0}=H_{0} \oplus E_{1}, \bar{E}_{0}=\bar{H}_{0} \oplus \bar{E}_{1} \text { with } E_{1} \perp H_{0}, \bar{E}_{1} \perp \bar{H}_{0} .
$$

$E_{1}$ and $\bar{E}_{1}$ are semisimple and they are infinite dimensional since they contain the infinite dimensional spaces $H_{1}$ and $\bar{H}_{1}$ respectively. Thus $E_{1}$ and $\bar{E}_{1}$ possess orthonormal bases and therefore

$$
E_{1} \cong \bar{E}_{1} \text {. }
$$

$E$ decomposes into orthogonal subspaces as follows

$$
E=(\operatorname{rad}(H) \oplus R) \oplus H_{0} \oplus E_{1}, E=(\operatorname{rad}(\bar{H}) \oplus \bar{R}) \oplus \bar{H}_{0} \oplus \bar{E}_{1}
$$

and this case is now completed as in case 1 .

Case 3. $H_{1}$ is finite dimensional. $E$ decomposes into orthogonal subspaces as in the second case:

$$
E=(\operatorname{rad}(H) \oplus R) \oplus H_{1} \oplus E_{2}, E=(\operatorname{rad}(\bar{H}) \oplus \bar{R}) \oplus \bar{H}_{1} \oplus \bar{E}_{2}, E_{2} \cong \bar{E}_{2}
$$

Let $F(F)$ be an algebraic complement of $H_{0}\left(\bar{H}_{0}\right)$ in $E_{2}\left(\bar{E}_{2}\right)$. If $H_{0}^{\perp_{0}}\left(\bar{H}_{0}^{\perp 0}\right)$ is the orthogonal complement of $H_{0}\left(\bar{H}_{0}\right)$ in $E_{2}\left(\bar{E}_{2}\right)$ we find that

$$
H_{0}^{\perp_{0}}=(0) \text { and } \bar{H}_{0}^{\perp_{0}}=(0) \text {. }
$$

Furthermore, $\operatorname{dim} F=\operatorname{dim} K+\operatorname{dim} L$ and $\operatorname{dim} F=\operatorname{dim} R+\operatorname{dim} L$. By (9) therefore

$$
\operatorname{dim}\left(E_{2} / H_{0}\right)=\operatorname{dim}\left(\bar{E}_{2} / \bar{H}_{0}\right) \text {. }
$$

In view of (11) and (12) we can apply the theorem quoted in 1.5: There exists an orthogonal isomorphism $T_{0}: E_{2} \rightarrow E_{2}$ with $T_{0}\left(H_{0}\right)=\bar{H}_{0}$. From isomorphisms (6) and (8) and decomposition (10) we thus secure an orthogonal automorphism of $E$ which maps $H=\operatorname{rad}(H) \oplus H_{0}$ onto $\bar{H}=\operatorname{rad}(\bar{H})+\bar{H}_{0}$. 
We are thus left with

Case 4. $H_{0}$ and $H_{1}$ are both infinite dimensional. We have

$$
E_{0}=H_{0} \oplus H_{1} \oplus L \oplus K, \bar{E}_{0}=\bar{H}_{0} \oplus \bar{H}_{1} \oplus L \oplus R \text { and } E_{0} \cong \bar{E}_{0} .
$$

In order to find an isomorphism $T_{0}: E_{0} \rightarrow \bar{E}_{0}$ which maps $H_{0}$ onto $\bar{H}_{0}$ we shall apply Theorem 1 to the space $E_{0}$. The orthogonal complement $H_{0}^{\perp_{0}}$ of $H_{0}$ in $E_{0}$ is precisely $H_{1}$, similarly $\bar{H}_{0}^{\perp_{0}}=\bar{H}_{1}$. Finally $H_{1}^{\perp_{0}}=H_{0} \oplus L$ and $\bar{H}_{1}^{\perp_{0}}=\bar{H}_{0} \oplus \bar{L}$. Hence

and

$$
\operatorname{dim}\left(H_{0}^{\perp_{0} \perp_{0}} / H_{0}\right)=\operatorname{dim} L=\operatorname{dim} \bar{L}=\operatorname{dim}\left(\bar{H}_{0}^{\perp_{0} \perp_{0}} / \bar{H}_{0}\right)
$$

$$
\operatorname{dim}\left(E_{0} /\left(H_{0}^{\perp_{0}}+H_{0}^{\perp_{0} \perp_{0}}\right)\right)=\operatorname{dim} K=\operatorname{dim} K=\operatorname{dim}\left(\bar{E}_{0} /\left(\bar{H}_{0}^{\perp_{0}}+\bar{H}_{0}^{\perp_{0} \perp_{0}}\right)\right) .
$$

Finally we recall that $H_{0}$ and $H_{1}$ are both trivially semisimple. Thus the assumptions of Theorem 2 are satisfied: there exists an orthogonal isomorphism $T_{0}: E_{0} \rightarrow \bar{E}_{0}$ with $T_{0}\left(H_{0}\right)=\bar{H}_{0}$. Together with the isomorphism $T_{1}$ of (b) we obtain an orthogonal isomorphism of $E$ which maps $H=\operatorname{rad}(H) \oplus H_{0}$ onto $\bar{H}=\operatorname{rad}(\bar{H}) \oplus \bar{H}_{0}$. This completes the proof of Theorem 2 .

If $\phi$ is a nondegenerate alternate form on the infinite dimensional space $E$ (1.3) then Lemmas 1 and 2, with the obvious modification that (iii) be dropped, hold for any commutative field whatsoever. For this reason the theorem quoted in 1.5 carries over to the alternate case for any arbitrary underlying field and the proof of Theorem 2 goes through mutatis mutandis (for example, $E_{0} \cong E_{0}$ in the last three cases because these spaces now possess symplectic bases and are of the same dimension). Furthermore, conditions on isomorphisms can now be replaced by simpler conditions on dimensions, as every semisimple space of at most denumerable dimension now admits of a sympletic basis. Thus, we obtain the following companion to Theorem 2:

THEOREM 3. Let $\phi$ be a nondegenerate, alternate form on the $k$-vectorspace $E$ of denumerable dimension, and $k$ an arbitrary commutative field (of any characteristic). Let $H$ and $\bar{H}$ be subspaces of $E$ with $\operatorname{rad}\left(H^{\perp}\right)=\operatorname{rad} H$, $\operatorname{rad}\left(\bar{H}^{\perp}\right)=\operatorname{rad}(H)$ and satisfying the following properties:

(i) $\operatorname{dim} H=\operatorname{dim} \bar{H}, \operatorname{dim}(\operatorname{rad} H)=\operatorname{dim}(\operatorname{rad} \bar{H})$,

(ii) $\operatorname{dim}(H / \operatorname{rad} H)=\operatorname{dim}(\bar{H} / \operatorname{rad} \bar{H})$,

(iii) $\operatorname{dim}\left(H+H^{\perp} / H\right)=\operatorname{dim}\left(\left(\bar{H}+\bar{H}^{\perp}\right) / \bar{H}\right)$,

(iv) $\operatorname{dim}\left(\left(H^{\perp}+H^{\perp \perp}\right) /\left(H^{\perp}+H\right)\right)=\operatorname{dim}\left(\left(\bar{H}^{\perp}+\bar{H}^{\perp \perp}\right) /\left(\bar{H}^{\perp}+\bar{H}\right)\right)$,

(v) $\operatorname{dim}\left((\operatorname{rad} H)^{\perp} /\left(H^{\perp}+H^{\perp \perp}\right)\right)=\operatorname{dim}\left((\operatorname{rad} \bar{H})^{\perp} /\left(\bar{H}^{\perp}+\bar{H}^{\perp \perp}\right)\right)$.

Then there exists a metric automorphism of $E$ which maps $H$ onto $\bar{H}$.

The last theorem partly confirms a conjecture by Kaplansky to the effect that equality of corresponding cardinal numbers defined by the subspaces $H$ and $\bar{H}$ is sufficient in the alternate case for an automorphism of the required kind to exist [4]. 


\section{BIBLIOGRAPHY}

1. N. Bourbaki, Formes sesquilinéaires et formes quadratiques, Hermann, Paris 1959.

2. H. Gross, Ein Wittscher Satz im Falle von Vektorräumen abzählbarer Dimension, J. Reine Angew. Math. 222 (1966), 195-200.

3. H. Gross and H. R. Fischer, Nonreal fields $k$ and infinite dimensional $k$-vectorspaces, Math. Ann. 159 (1965), 285-308.

4. I. Kaplansky, Forms in infinite dimensional spaces, An. Acad. Brazil. Ci. 22 (1950), 1-17.

\section{Montana State University,}

Bozeman, Montana 